\title{
Sum of Diameters of Non Lymph Node Tumors
}

National Cancer Institute

\section{Source}

National Cancer Institute. Sum of Diameters of Non Lymph Node Tumors. NCI

Thesaurus. Code C103441.

A calculation of the aggreg ated diameter values for tumors other than the lymph nodes. 